\title{
Predicting Sensory Quality of Longjing Tea on the Basis of Physiochemical Data
}

\author{
Ruicong Zhi ${ }^{1,2, *}$, Lei Zhao ${ }^{1}$, Bolin $\mathrm{Shi}^{1}$, Houyin Wang ${ }^{1}$, \\ Zhi Li ${ }^{1}$, Jian Zhang ${ }^{1}$, Xingjun $\mathrm{Xi}^{1}$ and $\mathrm{Yi} \mathrm{Jin}^{2}$ \\ ${ }^{1}$ Food and Agriculture Standardization Institute, China National Institute of Standardization, \\ Beijing 100088, China \\ ${ }^{2}$ Institute of Information Science, Beijing Jiaotong University, Beijing 100044, China
}

(Received May 7, 2012; accepted July 25, 2012)

Key words: sensory evaluation, instrumental measurement, artificial neural network, tea color, tea aroma, tea taste

Sensory evaluation can provide integrated, direct measurements of the perceived quality of food products. However, a sensory panel is subjective and suffers from inconsistency and inaccuracy. In this paper, we propose a sensory evaluation simulation model for Longjing tea (a Chinese brand of green tea). The physiochemical quality indicators of Longjing tea were determined by instrumental analysis, including color, aroma, and taste. Meanwhile, the sensory quality of the tea was evaluated by an expert sensory panel. An artificial neural network was conducted to approximately predict sensory evaluation scores on the basis of physiochemical data. The results showed that physiochemical factors, including hue, fluorescence peak 5, hue chromascale, b, L, 3-(methylthio) propionaldehyde, $\alpha$-terpineol, linalool, dimethyl sulfide, total aroma value, caffeine, quinic acid, theanin, gallic acid and total catechins were best correlated with sensory evaluation scores. Furthermore, physiochemical features that were chosen according to important factor weights were used to classify Longjing tea into two grades. Experimental results demonstrated that instrumental analysis could be complementarily used in the evaluation and control of sensory quality by establishing a reasonable sensory-instrument correlation and human-simulated predictive model.

\section{Introduction}

Chinese tea is an extensively consumed beverage worldwide with an expanding market. In addition, tea is one of the first set of medicinal herbs documented in ancient Chinese medicinal literature. (1) Quantification of tea quality is a difficult task because of the presence of innumerable compounds and their diverse contribution to tea quality. ${ }^{(2)}$ The major quality attributes of tea are appearance, color, aroma, and flavor. Moreover, tea quality is linked to the climate of the planting area and the manufacturer's specification. ${ }^{(3)}$

${ }^{*}$ Corresponding author: e-mail: zhirc_research@hotmail.com 
Evaluation of tea attributes is usually carried out by experienced tea tasters who have developed a language of their own, which is sometimes difficult to be understood by consumers, to describe various quality attributes of tea infusions. ${ }^{(4)}$ Gradation of tea is usually carried out according to the marks that are given by these tasters on a scale of 1 to 10, separately for appearance, aroma, and taste of the tea sample. ${ }^{(5)}$ Sensory evaluation can provide integrated, direct measurements of perceived intensities of tea attributes. However, tea tasters should be selected and trained carefully, and this process is laborious and time-consuming. Furthermore, human panel tasting is purely subjective and suffers from inconsistency and inaccuracy owing to various human factors such as individual variability, decrease in sensitivity due to prolonged exposure, fatigue, and variable mental states. ${ }^{(6)}$

Therefore, the development of methods that can partially replace the panel during routine work, achieving objective measurements in a short time and in a consistent and cost-effective manner is of great importance for the food industry. ${ }^{(7-9)}$ Presently, the chemical compounds related to tea flavor are tested using analytical instruments, such as gas chromatography (GC), ${ }^{(10,11)}$ high-performance liquid chromatography (HPLC), ${ }^{(12,13)}$ capillary electrophoresis $(\mathrm{CE}),{ }^{(14)}$ and plasma atomic emission spectrometry. ${ }^{(15)}$ Some researchers also reported the relationship between tea sensory evaluation and instrumental measurements, mainly using correlation coefficient. ${ }^{(16-19)}$ Lu et al. investigated the relationship between liquor chromaticity data and organoleptic quality of green tea, Oolong tea, and black tea using correlation coefficient and regression analysis. The results showed that the chromaticity data were significantly different between various types of tea and correlated with the sensory evaluation scores of tea. ${ }^{(16)}$ Zhao et al. analyzed the correlation between six biochemical components in tea, which was packed in several typical package materials to maintain freshness, and its sensory evaluation scores by multiple linear regression. The results showed that there was a close relationship between the oxidative degree of ascorbic acid and tea sensory evaluation scores, which was at the significant level of 0.997.(17) Linear regression was also used to regress components' data (the individual theaflavin composition, total theaflavins, thearubigins, theaflavin digallate equivalent, total color, and brightness) against sensory evaluation scores of two tasters. The theaflavin digallate equivalent showed the strongest relationship. ${ }^{(18)}$ A comparative study investigated stepwise multiple linear regression (MLR) analysis, principal component regression (PCR), and partial least squares (PLS) regression analysis, which were applied to the sensory evaluation scores and 77 GC peaks. Results showed that the sensory properties of tea aromas were statically correlated with the GC profiles of volatile flavor components. ${ }^{(19)}$ Moreover, some researchers have accomplished a study on the correlation between tea taster's sensory score and electronic nose/electronic tongue. . $^{(5,20,21)}$ Different pattern recognition models based on artificial neural network (ANN) were investigated to carry out a correlation study with the tea tasters' score. Encouraging performances were obtained with good accuracy. It showed the effectiveness of an adaptive model for estimating the sensory evaluation scores using an electronic nose and electronic tongue. However, there had been very few reports where physiochemical data has been used for the correlation with tea taster's evaluation results and selection of better physiochemical features for classification. Moreover, few studies focused on the relationship between different sensory attributes and instrumental measurements by using a human-simulated model. 
The aim of this study is to predict Longjing tea sensory quality according to physiochemical data by conducting ANN, and also to determine the physiochemical factors that are best correlated with sensory evaluation scores. Three types of sensory attributes were considered to show the relationship between sensory evaluation scores and physiochemical data, namely, color, aroma, and taste. The physiochemical data with high important factor weights were chosen as features to better represent tea samples. Furthermore, tea samples with different quality grades were identified by utilizing neural network. Recognition accuracies obtained using the selected physiochemical features were higher than that obtained using original physiochemical data.

\section{Materials and Methods}

\subsection{Tea samples and regents}

Tea samples: Longjing tea samples were selected from four well-known tea companies, including two grade levels (grade 1 and grade 2), two species (Longjing 43 and group species), and five geographical origins (Weng Jiashan, Ma Anshan, Yang Meiling, Mei Jiawu, and Long Menkan). The tea samples were numbered accordingly; the details of the tea samples are shown in Table 1. The aroma of tea samples is easily affected; therefore, all the samples were packed separately with aluminum foil and with silica gel desiccant in each pack. Then, the tea samples were sealed with a vacuum packing machine and stored at $4^{\circ} \mathrm{C}$ before testing. (22)

Regents: The 4-terpenols, geraniol, 1-octen-3-ol, linalool oxide, nerolidol, $\alpha$-terpineol, $\gamma$-terpinene, $\beta$-pinene, limonene, geraniol, $\beta$-ionone, 1-hexanol, heptanal, 3-(methylthio) propionaldehyde, $\beta$-myrcene, $\beta$-ocimene, linalylalcohol and 2-careen (Sigma-Aldrich, Shanghai, China, purity $>98 \%$ ) were diluted for training the GC-O panel.

Ethyl decylate (Sigma-Aldrich, Shanghai, China, purity $>99 \%$ ) was used as internal standard. The C8-C24 n-alkane mixture (Sigma-Aldrich, Shanghai, China, purity $>99 \%$ ) was injected after the sample under the same chromatographic condition to determinate LRI Kovat's index.

Epicatechin (EC), epicatechin-3-gallate (ECG), epigallocatechin-gallate (EGCG), catechin (C), gallocatechin-gallate (GCG), chlorogenic acid, gallic acid (GA), quinic acid, caffeic acid, caffeine, theanin, and vitamin C (VC) (Sigma-Aldrich, Shanghai, China, purity $>99 \%$ ) were used for taste analysis as HPLC external standard.

HPLC-grade acetonitrile and methanol were purchased from Fisher Reagent Company.

Table 1

Information of Longjing tea samples.

\begin{tabular}{lcccc}
\hline Variety & Species & Geographical origin & Grade level & Year \\
\hline Longjing & Longjing 43 (L) & WengJiashan (W) & $1(\mathrm{~T})$ & 2010 \\
& Group species (Q) & Ma Anshan (M) & $2(\mathrm{Y})$ & \\
& & Yang Meiling (Y) & & \\
& Mei Jiawu (Z) & & \\
\hline
\end{tabular}




\subsection{Sensory evaluation}

Tea samples were prepared as $3.0 \mathrm{~g}$ of tea leaves, infused with $150 \mathrm{~mL}$ of distilled boiling water for $5 \mathrm{~min}$. By using a sieve, the tea infusion was transfered to glasses, and the leaves were left. The tea was evaluated on the basis of shape, color, aroma, taste, and leaves. Sensory evaluation procedure was conducted in accordance with the Chinese National Standard as GB/T 23776-2009 Methodology of Sensory Evaluation of Tea. Seven senior tea tasters (specialized expert assessors) were chosen to form a panel. Panelists evaluated the five attributes (shape, color, aroma, taste, and leaves) for each test sample by giving a score within 100 following the standard evaluation procedure. The result of each tea sample for each attribute was the average score given by all the panelists. The final score of each tea sample was calculated by taking account of attribute factor weights, which are shown in Table 2 as indicated in GB/T 23776-2009 Methodology of Sensory Evaluation of Tea.

\subsection{Instrumental measurements}

\subsubsection{Color}

Sample preparation: $1.0 \mathrm{~g}$ of Longjing tea sample was stirred with $100 \mathrm{~mL}$ of boiling water for $30 \mathrm{~min}$, then cooled to room temperature $\left(22^{\circ} \mathrm{C}\right)$. Each tea sample was measured parallel three times. The average value was taken for data analysis.

Fluorescence spectroscopy measurement: Three-dimensional fluorescence scanning with F-7000 Fluorescence spectrometer (Hitachi Limited) was carried out for the tea samples, so that spectrograms were obtained. Five fluorescence peaks were selected from the spectrograms with different excitation wavelengths $(300-550 \mathrm{~nm})$ and emission wavelengths $(310-750 \mathrm{~nm})$.

Chromatic aberration measurement: A Color Quest XE Colorimeter (Hunter Lab) was used to measure chromatic aberration for the tea samples, and the corresponding chroma spectral data was obtained. There were five chromatic aberration factors, namely, L, a, b, hue, and hue chromascale. ${ }^{(23)}$

\subsubsection{Aroma}

Sample preparation: $10.0 \mathrm{~g}$ of Longjing tea sample was stirred with $50 \mathrm{~mL}$ of boiling water (ultrapure) and covered to keep warm for $15 \mathrm{~min}$, and then the tea leaves were filtered off. The tea infusion was transferred to a $20 \mathrm{~mL}$ headspace bottle, which had sodium chloride (1.9 g, analytically pure). $1 \mu \mathrm{L}$ of internal standard solution (ethyl caprate, $0.1 \mu \mathrm{L} / 1 \mathrm{~mL}$ in methanol) was loaded into a microliter syringe, and then stamped sealed.

Equipment conditions: To keep the original aroma of Longjing tea, dynamic headspace sampling (ITEX) technology was applied (CTC Analytics AG, Switzerland) to absorb sample top gas and recycle enrichment. The technical principle of ITEX is shown in

Table 2

Factor weights of five tea attributes.

\begin{tabular}{lccccc}
\hline & Shape & Color & Aroma & Taste & Leaves \\
\hline Factor weights & 0.30 & 0.10 & 0.25 & 0.25 & 0.10 \\
\hline
\end{tabular}


Fig. 1, and the parameters of ITEX are listed in Table 3. After separating by capillary chromatography, mass spectrometry analysis and odor sniffing were carried out and the results were analyzed simultaneously with a split ratio of 1:1. Each sample was analyzed two times, and the average value was adopted.

A DB-5MS column $(30 \mathrm{~m} \times 0.25 \mathrm{~mm}$ i.d., $0.25 \mu \mathrm{m}$ film thickness $)$ was used for the analyses. The temperature program used was as follows: $40^{\circ} \mathrm{C}$ for $2 \mathrm{~min}$ and then was raised to $180^{\circ} \mathrm{C}$ at $4^{\circ} \mathrm{C} / \mathrm{min}$, and to $245^{\circ} \mathrm{C}$ at $8^{\circ} \mathrm{C} / \mathrm{min}$ final isotherm for $5 \mathrm{~min}$. The carrier gas was helium $(99.99 \%$ ) at a flow rate of $1.5 \mathrm{~mL} / \mathrm{min}$. The MS detector operated in the scan mode with $70 \mathrm{eV}$ electron impact, scanning the $45-300 \mathrm{~m} / \mathrm{z}$ range. The multiplier was at $1.0 \mathrm{kV}$; the source temperature and transfer lines were at 230 and $280^{\circ} \mathrm{C}$, respectively. ${ }^{(24)}$

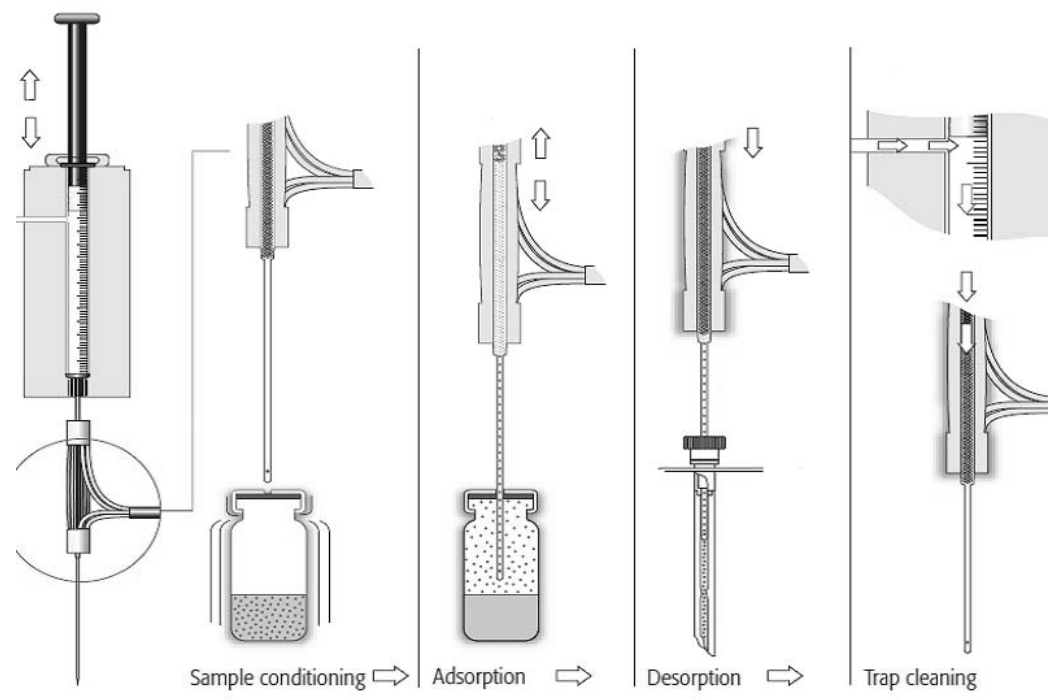

Fig. 1. Technical principle of ITEX.

Table 3

Parameters list of ITEX.

\begin{tabular}{lr}
\hline Parameter & Value \\
\hline Incubator oven temperature $\left({ }^{\circ} \mathrm{C}\right)$ & 80 \\
I Incubation time $(\mathrm{s})$ & 1200 \\
Agitator speed $(\mathrm{rpm})$ & 500 \\
needle temperature $\left({ }^{\circ} \mathrm{C}\right)$ & 85 \\
Extraction volume $(\mu \mathrm{L})$ & 2000 \\
Extraction strokes & 100 \\
Extraction speed $(\mu \mathrm{L} / \mathrm{s})$ & 200 \\
Desorption temperature $\left({ }^{\circ} \mathrm{C}\right)$ & 230 \\
Desorption speed $(\mu \mathrm{L} / \mathrm{s})$ & 20 \\
Flush needle tempreture $\left({ }^{\circ} \mathrm{C}\right)$ & 250 \\
Flush needle time $(\mathrm{s})$ & 300 \\
\hline
\end{tabular}


Characteristic aroma compound analysis: There are three main steps in the analysis of Longjing tea's characteristic aroma compounds: 1) determine Longjing tea's aroma compounds qualitatively using mass spectrometry (NIST08 mass spectral library), relative reservation index (RI), and sniffing method; 2) utilizing ethyl decanoate as internal standard, detect the main qualitative aroma compounds quantitatively; 3) determine the characteristic aroma compounds of Longjing tea based on GC-O technique (Agilent 7890-5975C, gas chromatograph-mass spectrometer (GC-MS), Agilent Technologies Company, U.S.; Sniffer 9000 (O, the sniffing detector), Brechbühler900 Company, Switzerland). The frequency detection method combined with the detection intensity method ${ }^{(25)}$ was used. The frequency detection method is easy to use, less timeconsuming, and not highly restricted to assessors. The result is reproducible and can reflect the sensitivity differences among assessors. ${ }^{(26)}$ In our experiment, the GC-O panel consists of five assessors, including 4 females and 1 male, aging from 20 to 32. All the assessors have passed the single sample training test of 17 types of aroma reference samples. Each assessor had to sniff a tea sample three times, and 3-scale method was applied to evaluate the tea sample. In total, each substance was sniffed 15 times. If a substance was recognized more than 9 times, and the intensity was evaluated bigger than 2 for more than 7 times, then it was considered to be the characteristic aroma compound of Longjing tea.

\subsubsection{Taste}

Sample preparation: $1.0 \mathrm{~g}$ of Longjing tea sample was stirred with $300 \mathrm{~mL}$ of boiling water; then, the beaker was placed in a boiling water bath for $20 \mathrm{~min}$, with shaking every $5 \mathrm{~min}$. The tea was pump-filtered under reduced pressure while hot, then cooled to room temperature, and filtered with a membrane $(0.45 \mu \mathrm{m})$.

Equipment conditions: A Venusil MP C18 column $(250 \mathrm{~mm} \times 4.6 \mathrm{~mm}, 5 \mu \mathrm{m})$ was used for the analyses. Mobile phase: acetonitrile (B) and $0.05 \%$ formic acid in water (A), using a gradient elution program: 0 to $10 \mathrm{~min}, \mathrm{~B}: 0-12 \%, 10$ to $40 \mathrm{~min}, \mathrm{~B}: 12-25 \%$; flow rate: $1 \mathrm{~mL} / \mathrm{min}$. The variable wavelength detector (VWD) was operated in $280 \mathrm{~nm}$. The Evaporative Light Scattering detector (ELSD) was set up as follows: temperature of drift tube is $95^{\circ} \mathrm{C}$, gas flow rate is 3.5 bar, and gain value is 6 .

Taste compound analysis: Firstly, we need to determine the high-performance liquid chromatography method for 12 types of taste compound, construct the working curve by external standard method, and carry out a systematic study on the performance of accuracy, recovery, and linear range. Agilent 1200 high-performance liquid chromatograph (HPLC), produced by Agilent Technologies Company, U.S., was used. The concentration of the sample solution was calculated on the basis of peak area of each compound. The average value was taken for data analysis.

\subsection{Data analysis and modeling}

ANN is a mathematical tool, which can make a nonlinear mapping between input and output spaces. The structure of ANN is somehow like a simulation of the human brain. It is pseudoparallel processing systems capable of "adaptable learning", meaning that they can do so without the formalisms and restrictions of computer programming languages. 
The multilayer perceptron (MLP) network is probably the most popular ANN in the case of nonlinear mapping. Normally, it consists of an input layer, a hidden layer, and an output layer. The schematic diagram of a three-layer ANN model is shown as Fig. 2. The input nodes receive the data values and pass them to nodes in the first hidden layer. Each node collects the input from all the input nodes after multiplying each input value by a weight, attaches a bias to this sum, and passes on the results through a nonlinear transformation like the sigmoid transfer function. This forms the input either for the second hidden layer or the output layer that operates identically to the hidden layer. The resulting transformed output from each output node is the network output. (27)

The fundamental constituents of the ANNs are called nodes or neurons. Figure 3 shows a basic representation of such neuron. Each neuron performs a series of simple calculation. The input data are processed by a neuron according to

$$
n e t_{i}=\sum_{i=1}^{n} w_{i} x_{i}
$$

where $w_{i}$ denotes the weights, an analogy of the biological synapses. $x_{i}$ and $n e t_{i}$ are the input and output of neurons, respectively. net $t_{i}$ may take large positive or negative values; therefore, it is further modified in a second step. The final output of the MLP network can be expressed as

$$
y=\sigma\left(\sum_{i=1}^{n} w_{i} x_{i}-\theta\right)=\sigma\left(\text { net }_{i}-\theta\right) .
$$

The term $\theta$ is a threshold value above which the neuron is said to "fire" or emit a signal. $\sigma(\bullet)$ is the activation function that restricts the output of the neuron to between 0 and 1 or -1 to 1 .

In this paper, MLP network was used to treat the data obtained from sensory evaluation and instrumental measurement. Instrumental data were utilized to predict

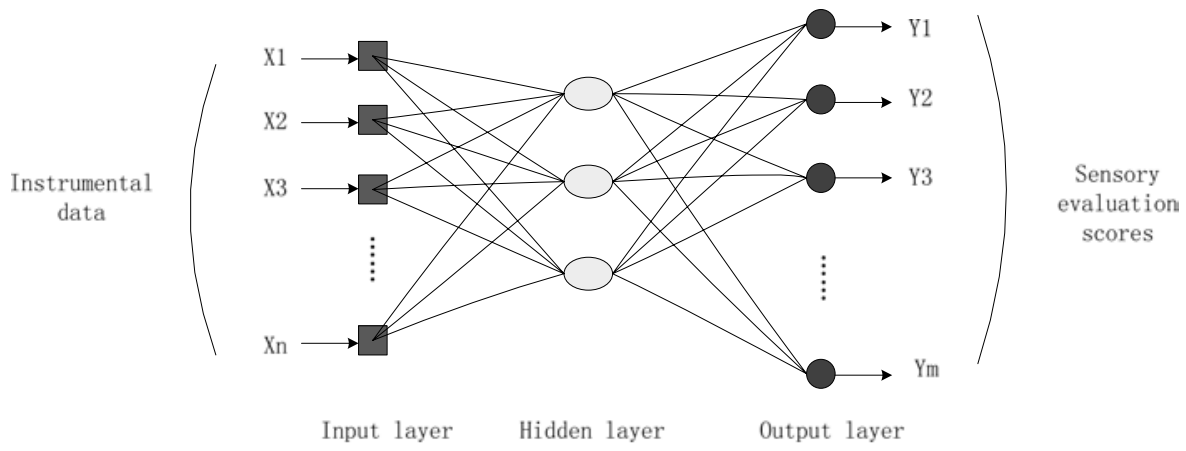

Fig. 2. Schematic diagram of MLP model. 


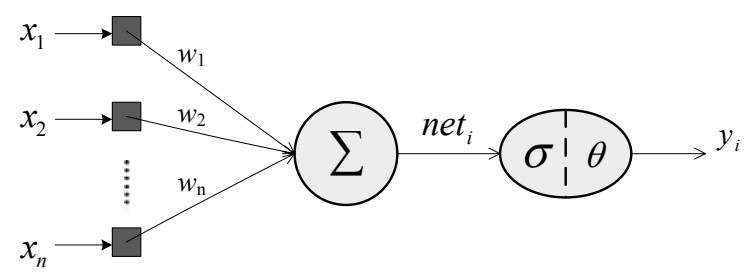

Fig. 3. Diagram of an artificial neuron.

tea-taster evaluation results. The input layer of ANN was fed with the output from instrumental measurement data, and the output layer was configured to show the tea taster's score. Training of the neural networks was performed with $80 \%$ of all the tea samples, and the remaining tea samples were used for testing.

The performance of MLP network can be evaluated by calculating the differences between real sensory evaluation value and prediction value. With this regard, the mean squared error (MSE) may be utilized, which is defined as

$$
M S E=\frac{\sum_{i}\left(d_{i}-o_{i}\right)^{2}}{n}
$$

where $d_{i}$ and $o_{i}$ represent the predicted sensory evaluation value and actual evaluation value, respectively. $n$ is the number of input patterns. The smaller the MSE value, the higher the prediction accuracy of the MLP network model.

\section{Results and Discussion}

\subsection{Sensory quality prediction}

\subsubsection{Sensory quality prediction utilizing color instrumental measurement}

Color instrumental data of the tea samples consist of fluorescence peak values and chromatic aberration values, which are partially shown in Table 4. These ten factors were treated as the input of ANN, while the sensory evaluation scores of color attribute were treated as the output of ANN. One hidden layer of the MLP network was chosen by experiments.

The residual of predictive value and actual sensory evaluation marks, i.e., residual values, are shown in Fig. 4. It can be seen that most of the absolute of residual values are within 0.5 ; comparing with the score of 100 , the error tolerance is around $0.5 \%$. The mean squared errors for training and testing are 0.25 and 1.90 , respectively.

Besides the predictive value, the ANN model also gives the weights of the ten color factors, as shown in Fig. 5. For example, the first five most important color factors of Longjing tea are hue, peak 5 , hue chromascale, $\mathrm{b}$, and $\mathrm{L}$, whose normalized factor weights are $1,0.83,0.72,0.64$, and 0.54 , respectively. 
Table 4

Color instrumental data of the tea samples.

\begin{tabular}{|c|c|c|c|c|c|c|c|c|c|c|}
\hline \multirow[b]{2}{*}{ No. } & \multicolumn{5}{|c|}{ Chromatic aberration } & \multicolumn{5}{|c|}{ Fluorescence } \\
\hline & $\bar{L}$ & $\mathrm{a}$ & $\mathrm{b}$ & hue & $\begin{array}{c}\text { Hue } \\
\text { chromascale }\end{array}$ & Peak 1 & Peak 2 & Peak 3 & Peak 4 & Peak 5 \\
\hline 1 & 33.93 & -0.69 & 1.31 & -1.88 & 1.48 & 403.6 & 68.87 & 73.62 & 75.66 & 65.61 \\
\hline 2 & 33.91 & -0.69 & 1.34 & -1.72 & 1.39 & 416.0 & 81.74 & 85.51 & 86.43 & 75.42 \\
\hline 3 & 34.21 & -0.59 & 0.99 & -1.70 & 1.29 & 342.2 & 68.57 & 73.14 & 76.23 & 64.83 \\
\hline 4 & 34.11 & -0.51 & 0.45 & -1.59 & 1.27 & 389.1 & 71.05 & 72.40 & 76.10 & 65.40 \\
\hline 5 & 34.01 & -0.78 & 1.48 & -1.56 & 1.25 & 317.1 & 75.22 & 79.01 & 82.04 & 71.49 \\
\hline 6 & 33.88 & -0.75 & 1.52 & -2.15 & 2.04 & 314.1 & 74.31 & 76.53 & 80.59 & 68.52 \\
\hline 7 & 34.02 & -0.53 & 0.79 & -1.70 & 1.40 & 377.7 & 64.40 & 62.77 & 62.93 & 52.47 \\
\hline 8 & 34.11 & -0.76 & 1.40 & -1.33 & 0.98 & 224.3 & 59.82 & 62.10 & 67.08 & 57.73 \\
\hline 9 & 33.94 & -0.61 & 1.15 & -1.88 & 1.30 & 538.1 & 66.25 & 64.29 & 68.62 & 57.28 \\
\hline 10 & 33.82 & -0.73 & 1.60 & -2.20 & 1.75 & 343.0 & 83.94 & 85.55 & 87.90 & 73.04 \\
\hline 11 & 34.08 & -0.69 & 1.03 & -2.90 & 1.92 & 380.1 & 64.18 & 64.65 & 65.32 & 57.04 \\
\hline 12 & 34.07 & -0.70 & 1.20 & -2.03 & 1.58 & 290.3 & 65.97 & 71.12 & 73.03 & 64.23 \\
\hline
\end{tabular}

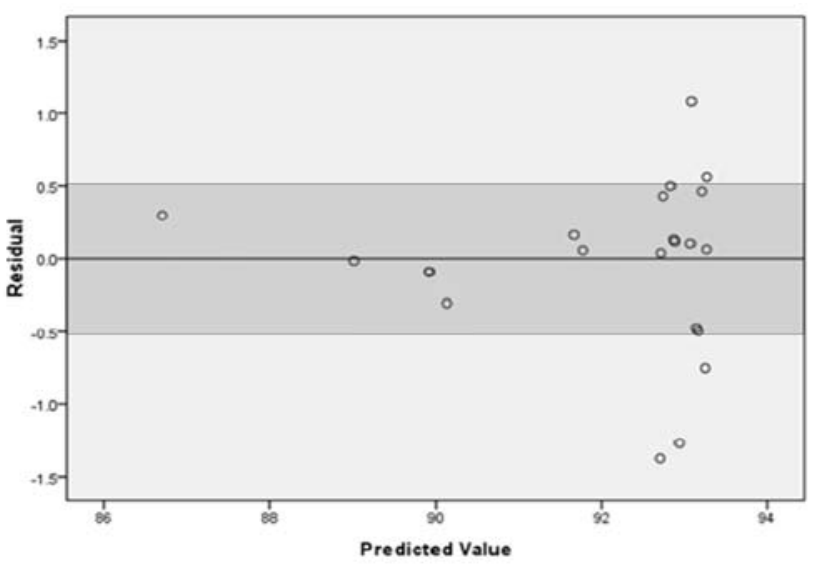

Fig. 4. Residual values of the ANN predicted value (color).

\subsubsection{Sensory quality prediction utilizing aroma instrumental measurement}

According to $\S 2.3 .2$, characteristic aroma compounds were obtained by the GCO-MS technique. The characteristic aroma compounds consist of dimethyl sulfide, 3-(methylthio) propionaldehyde, (3-ethyl-2, 5-dimenthylpyrazine), linalool, $\alpha$-terpineol, geraniol, cis-jasmone, and the total value of the previous seven compounds, which are partially shown in Table 5. These eight factors were treated as the input of ANN, while the sensory evaluation scores of aroma attribute were treated as the output of ANN. One hidden layer of the MLP network was chosen by experiments.

The residual of predictive value and actual sensory evaluation marks are shown in Fig. 6. It can be seen that most of the absolute of residual values are within 1; comparing with 


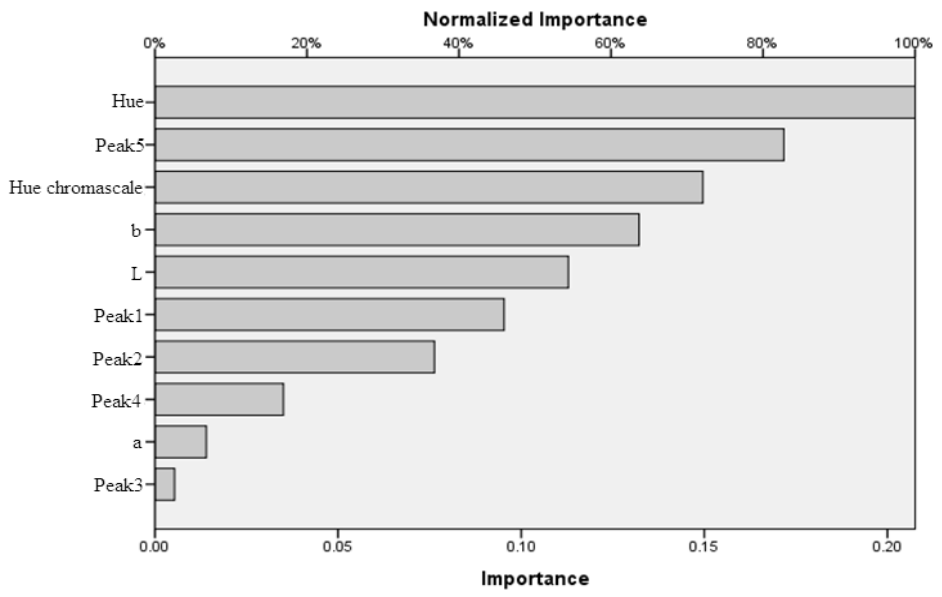

Fig. 5. Important factor weights of color instrumental measurement.

Table 5

Aroma instrumental data of the tea samples.

\begin{tabular}{|c|c|c|c|c|c|c|c|c|}
\hline No. & $\begin{array}{l}\text { Dimethyl } \\
\text { suilfide }\end{array}$ & $\begin{array}{c}3- \\
\text { (methylthio) } \\
\text { propional } \\
\text { dehyde }\end{array}$ & $\begin{array}{c}\text { 3-ethyl- } \\
2,5- \\
\text { dimenthyl } \\
\text { pyrazine }\end{array}$ & Linalool & $\begin{array}{c}\alpha- \\
\text { terpineol }\end{array}$ & Geraniol & $\begin{array}{c}\text { Cis- } \\
\text { jasmone }\end{array}$ & Total \\
\hline 1 & 148.27 & 3.49 & 21.18 & 792.91 & 234.36 & 156.07 & 5.35 & 1361.63 \\
\hline 2 & 81.51 & 1.64 & 19.47 & 582.02 & 95.41 & 64.79 & 1.71 & 846.55 \\
\hline 3 & 89.69 & 4.88 & 32.92 & 782.24 & 210.85 & 192.9 & 3.95 & 1317.43 \\
\hline 4 & 216.64 & 2.16 & 18.88 & 568.41 & 113.98 & 90.76 & 2.69 & 1013.52 \\
\hline 5 & 195.86 & 2.06 & 22.76 & 768.44 & 238.89 & 143.31 & 1.84 & 1373.26 \\
\hline 6 & 121.72 & 3.55 & 22.56 & 948.31 & 220.75 & 125.06 & 1.69 & 1443.64 \\
\hline 7 & 61.83 & 3.46 & 21.58 & 1418.68 & 257.76 & 194.98 & 31.17 & 1989.46 \\
\hline 8 & 61.45 & 0 & 20.80 & 1150.23 & 283.40 & 208.58 & 8.62 & 1733.08 \\
\hline 9 & 62.07 & 2.49 & 16.97 & 721.36 & 237.40 & 154.74 & 2.73 & 1197.76 \\
\hline 10 & 236.1 & 5.24 & 44.91 & 757.49 & 243.90 & 208.05 & 2.99 & 1498.68 \\
\hline 11 & 81.12 & 4.07 & 21.49 & 813.45 & 261.53 & 153.91 & 2.65 & 1338.22 \\
\hline 12 & 98.26 & 2.48 & 5.17 & 461.64 & 66.88 & 78.82 & 1.60 & 714.85 \\
\hline
\end{tabular}

the score of 100 , the error tolerance is around $1 \%$. The mean squared errors for training and testing are 2.35 and 0.45 , respectively.

Besides the predictive value, the ANN model also gives the weights of the eight aroma factors, as shown in Fig. 7. For example, the first five most important aroma factors of Longjing tea are 3-(methylthio) propionaldehyde, total aroma value, alphaterpineol, linalool, and dimethyl sulfide, whose normalized factor weights are 1, 0.25, 0.23, 0.22 , and 0.65 , respectively. 


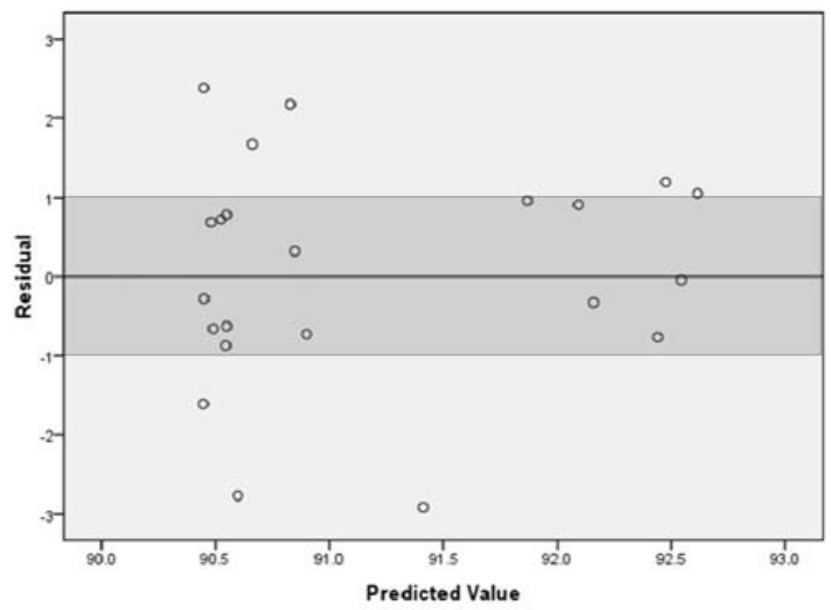

Fig. 6. Residual values of the ANN predicted value (aroma).

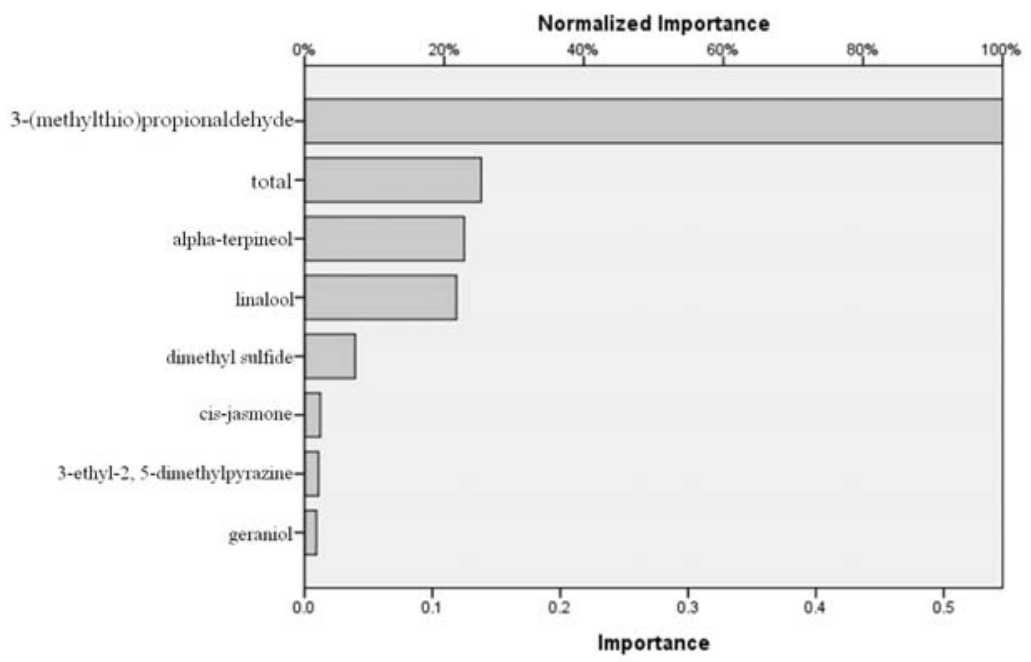

Fig. 7. Important factor weights of aroma instrumental measurement.

\subsubsection{Sensory quality prediction utilizing taste instrumental measurement}

According to $\S 2.3 .3$, taste compounds of Longjing tea were obtained by highperformance liquid chromatography (HPLC). These taste compounds consist of quinic acid, VC, theanin, caffeine, GA, chlorogenic acid, cafeic acid, EC, C, GCG, ECG, EGCG, and total catechins. Parts of the original taste instrumental data are shown in Table 6. These thirteen factors were treated as the input of ANN, while the sensory 
Table 6

Taste instrumental data of the tea samples.

\begin{tabular}{|c|c|c|c|c|c|c|c|c|c|c|c|c|c|}
\hline & $\begin{array}{c}\text { Quinic } \\
\text { acid }\end{array}$ & $\mathrm{VC}$ & Theanin & Caffeine & GA & $\begin{array}{c}\text { Chlorogenic } \\
\text { acid }\end{array}$ & $\begin{array}{c}\text { Cafeic } \\
\text { acid }\end{array}$ & $\mathrm{EC}$ & $\mathrm{C}$ & GCG & ECG & EGCG & Total \\
\hline 1 & 1.18 & 3.89 & 15.57 & 29.69 & 3.48 & 2.51 & 2.28 & 7.59 & 17.10 & 10.90 & 35.27 & 81.13 & 151.99 \\
\hline 2 & 0.89 & 2.91 & 11.63 & 29.29 & 2.40 & 2.68 & 3.59 & 11.97 & 16.07 & 16.73 & 36.82 & 84.68 & 166.26 \\
\hline 3 & 0.84 & 3.62 & 14.47 & 31.00 & 3.61 & 2.71 & 2.58 & 8.60 & 19.03 & 13.73 & 40.13 & 92.29 & 173.79 \\
\hline 4 & 0.82 & 2.83 & 11.31 & 27.07 & 3.18 & 3.21 & 3.01 & 10.02 & 21.33 & 11.72 & 29.94 & 68.87 & 141.89 \\
\hline 5 & 1.08 & 2.87 & 11.47 & 28.10 & 3.11 & 3.75 & 2.80 & 9.32 & 19.55 & 17.56 & 34.00 & 78.21 & 158.64 \\
\hline 6 & 0.79 & 3.43 & 13.71 & 29.91 & 2.75 & 2.57 & 2.64 & 8.80 & 17.83 & 15.15 & 35.19 & 80.93 & 157.90 \\
\hline 7 & 0.89 & 3.46 & 13.86 & 28.47 & 3.18 & 2.73 & 3.65 & 12.18 & 17.30 & 27.98 & 32.14 & 73.93 & 163.53 \\
\hline 8 & 1.39 & 4.64 & 18.58 & 28.85 & 2.85 & 3.61 & 2.87 & 9.56 & 21.72 & 15.32 & 32.86 & 75.57 & 155.03 \\
\hline 9 & 0.57 & 3.40 & 13.61 & 30.21 & 2.93 & 4.16 & 5.67 & 18.91 & 11.85 & 13.08 & 40.70 & 93.60 & 178.14 \\
\hline 10 & 0 & 3.51 & 14.05 & 31.31 & 3.37 & 3.53 & 3.97 & 13.25 & 20.42 & 11.57 & 34.70 & 79.82 & 159.77 \\
\hline 11 & 0.55 & 2.74 & 10.98 & 26.85 & 2.47 & 3.85 & 2.67 & 8.92 & 23.57 & 11.77 & 33.14 & 76.22 & 153.63 \\
\hline 12 & 1.03 & 3.78 & 15.12 & 30.85 & 3.04 & 2.99 & 2.81 & 9.36 & 21.70 & 14.68 & 39.28 & 90.33 & 175.34 \\
\hline
\end{tabular}

evaluation scores of taste attribute were treated as the output of ANN. One hidden layer of the MLP network was chosen by experiments.

The residual of predictive value and actual sensory evaluation marks are shown in Fig. 8. It can be seen that most of the absolute of residual values are within 1; comparing with the score of 100 , the error tolerance is around $1 \%$. The mean squared errors for training and testing are 1.04 and 0.53 , respectively.

Besides the predictive value, the ANN model also gives the weights of the thirteen taste factors, as shown in Fig. 9. For example, the first five most important taste factors of Longjing tea are caffeine, quinic acid, theanin, gallic acid, and total catechins, whose normalized factor weights are $1,0.55,0.28,0.22$, and 0.19 , respectively.

\subsection{Classification for tea grade}

In this section, ANN was used to identify the grade of tea samples. The selection of ANNs for classification of tea samples was conducted, considering the reduction in the mean square error for various networks' topologies. Figure 10 shows the trend of MSE for the ANN. In order to avoid the overtraining problem, the network training was thus stopped after 1000 cycles.

The physiochemical data was treated as the input of ANN, while the grade of tea samples was treated as the output of ANN. One hidden layer of the MLP network was chosen. In our experiment, the "leave-one-out" strategy ${ }^{(28)}$ was utilized to validate the performance of grade classification for tea samples. That is, one tea sample was removed from the sample set and all the remaining tea samples were used for training. The same procedure was repeated 28 times, and the average accuracy was recorded.

The factors of color, aroma, and taste are ranked according to the important weights, as shown in $\S \S 3.1 .1,3.1 .2$, and 3.1.3. The value of important weights denotes the influence capability of each factor. There is no borderline to determine which features 


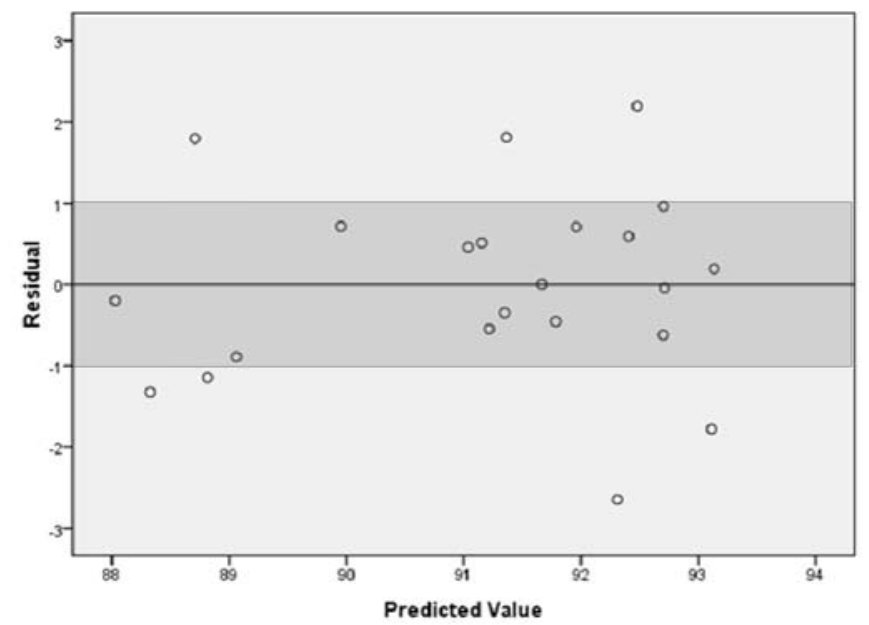

Fig. 8. Residual values of the ANN predicted value (taste).

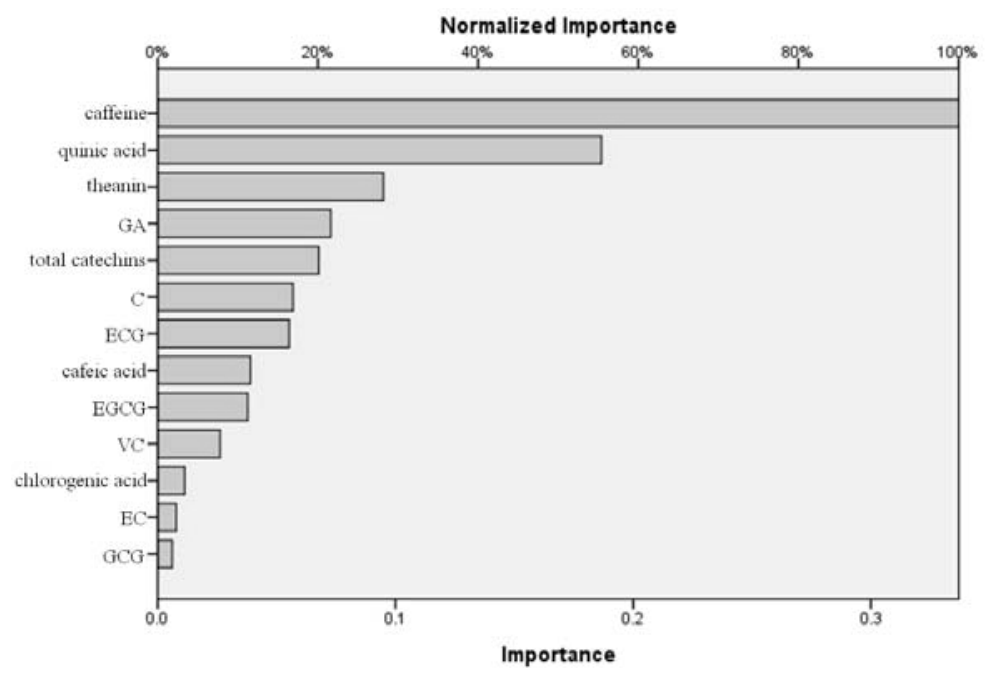

Fig. 9. Important factor weights of taste instrumental measurement.

should be chosen. The physiochemical features are selected according to the basis of factor weights (in descending order). Comparison of classification performances for different numbers of selected physiochemical features with original physiochemical features is shown in Fig. 11, for class 1, class 2, and the total results.

It can be seen from the illustration that the recognition accuracies were changing versus selected feature numbers. The trend of the curve shows that the top recognition 


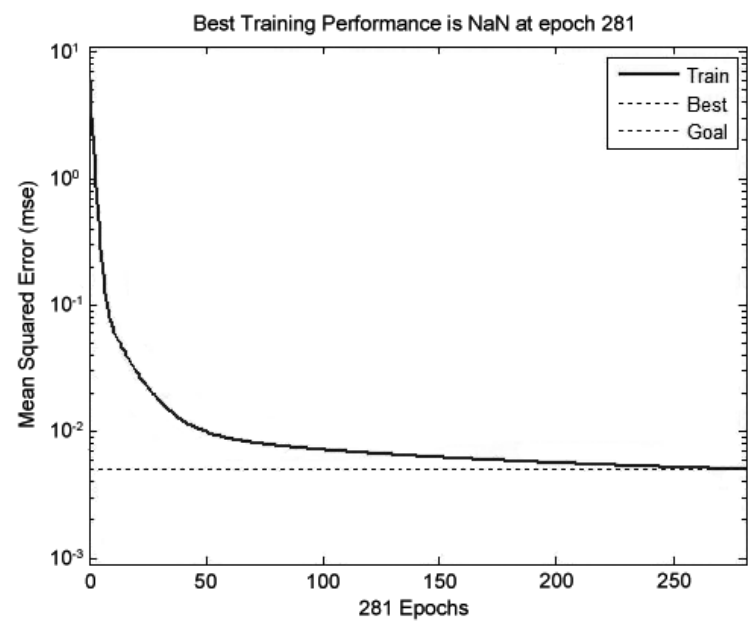

Fig. 10. Training MSE for ANN.

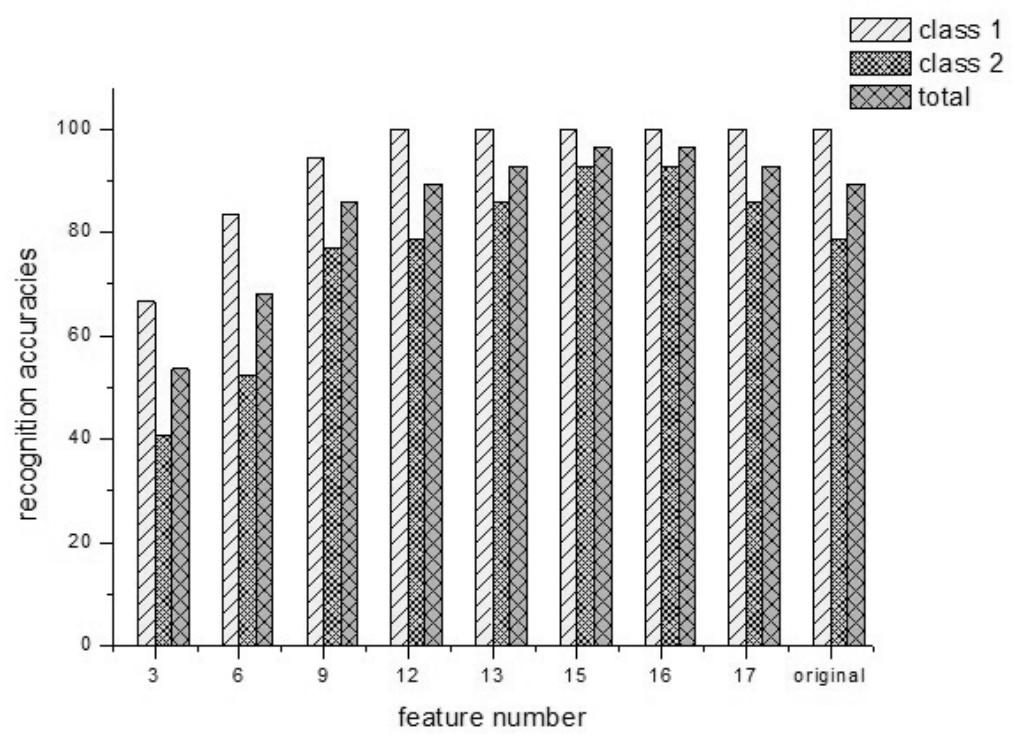

Fig. 11. Comparison of classification results (\%) of original physiochemical data and different number of selected physiochemical features.

accuracy does not appear in the largest feature dimensions (i.e., original features). When the selected physiochemical feature numbers reduced to 12 , the recognition accuracies were higher than that of original features, especially when the feature number was 15 . 
These fifteen selected physiochemical features were hue, peak 5 , hue chromascale, b, L, 3-(methylthio)propionaldehyde, total aroma value, $\alpha$-terprineol, linalool, dimethyl sulfide, caffeine, quinic acid, theanin, gallic acid, and total catechins. For the fifteen selected physiochemical features, the recognition accuracy of class one is $100 \%$, which is the same as that of unselected data. Whereas the recognition accuracy for class two with selected physiochemical features is $92.8 \%$, much higher than that of unselected data, which is $78.6 \%$. In total, the selected physiochemical features can better distinguish Longjing tea samples with two grades. The average recognition accuracies obtained by two types of input are $96.4 \%$ and $89.3 \%$, respectively. As sensory attributes with high important factor weights represent the tea samples efficiently, the selected physiochemical features can achieve the same or even higher recognition accuracies than that of the original physiochemical data, while the number of tea instrumental factors is much smaller. Therefore, it is efficient to select physiochemical features that highly affect the tea sensory quality to improve the classification results.

\section{Conclusion}

In this paper, we proposed a sensory evaluation simulation model for Longjing tea (a brand of green tea). Sensory evaluation results were approximately predicted using three types of instrumental data (color, aroma, and taste), and the physiochemical factors that best correlated with sensory evaluation were determined.

The major aim of our study is to develop a taster-specific computational model for the objective prediction of tea quality using instrumental measurement. Several interesting items should be pointed out:

(1) By using the ANN model, sensory evaluation could be predicted by instrumental measurement efficiently. The relationship between sensory evaluation and instrument measurement was investigated for three types of tea attribute, i.e., color, aroma, and taste.

(2) Physiochemical factors with high weights were selected, which means that these factors were best correlated with sensory evaluation results.

(3) The selected physiochemical features could be used for Longjing tea classification so as to reduce the computational complexity and enhance the recognition accuracy. ANN was used to classify Longjing tea samples with two grades, and higher recognition accuracies were reached by the selected physiochemical features.

Results from these analyses demonstrate that instrumental measurement could be used in sensory quality evaluation and control by establishing reasonable sensoryinstrument correlation and human-simulated predictive model.

\section{Acknowledgements}

This work was supported by the National Natural Science Foundation of China (No. 31201358), and China National High Technology Research and Development Program 863 (No. 2011AA1008047). 


\section{References}

1 S. Nie, M. Xie, Z. Fu, Y. Wan and A. Yan: Carbohydr. Polym. 71 (2008) 626.

2 B. Banerjee: Tea: Production and Processing (Oxford \& IBH, New Delhi, 1993).

3 S. Tian, S. Deng and Z. Chen: Sens. Actuators, B 123 (2007) 1049.

4 Y. Liang, J. Lu, L. Zhang, S. Wu and Y. Wu: Food Chem. 80 (2003) 283.

5 N. Bhattacharyya, R. Bandyopadhyay, M. Bhuyan, B. Tudu, D. Ghosh and A. Jana: IEEE Trans. Instrum. Meas. 57 (2008) 1313.

6 R. Dutta, E. L. Hines, J. W. Gardner, K. R. Kashwan and M. Bhuyan: Sens. Actuators, B 94 (2003) 228.

7 M. Scampicchio, S. Benedetti, B. Brunetti and S. Mannino: Electroanalysis 18 (2006) 1643.

8 Y. Uchiyama, M. Yamashita, M. Kato, T. Suzuki, M. Omori and R. Chen: Sens. Mater. 23 (2011) 501.

9 N. Hayashi, R. Chen, H. Ikezaki and T. Ujihara: J. Agric. Food Chem. 56 (2008) 7384.

10 N. Togari, A. Kobayashi and T. Aishima: Food Res. Int. 28 (1995) 495.

11 L. Wang, J. Lee, J. Chung, J. Baik and S. Park: Food Chem. 109 (2008) 196.

12 M. Pelillo, B. Biguzzi, A. Bendini, T. GallinaToschi, M. Vanzini and G. Lercker: Food Chem. 78 (2002) 369.

13 Y. G. Zou, H. Chen and Y. W. Deng: Talanta 57 (2002) 307.

14 H. Horie, T. Mukai and K. Kohata: J. Chromatogr. A 758 (1997) 332.

15 M. A. Herrador and A. G. Gonzalez: Talanta 53 (2001) 1249.

16 J. Lu, Y. Liang, S. Gong, Z. Gu, L. Zhang and Y. Xu: J. Tea Sci. 22 (2002) 57.

17 S. Zhao and X. Liu: J. Anhui Agric. Sci. 38 (2010) 6197.

18 P. O. Owuor, M. Obanda, H. E. Nyirenda, N. I. K. Mphangwe, L. P. Wright and Z. Apostolides: Food Chem. 97 (2006) 644.

19 N. Togari, A. Kobayashi and T. Aishima: Food Res. Int. 38 (1995) 485.

20 B. Tudu, A. Jana, A. Metla, D. Ghosh, N. Bhattacharyya and R. Bandyopadhyay: Sens. Actuators, B 138 (2009) 90.

21 M. Palit, B. Tudu, P. K. Dutta, A. Dutta, A. Jana, J. K. Roy, N. Bhattacharyya, R. Bandyopadhyay and A. Chatterjee: IEEE Trans. Instrum. Meas. 59 (2010) 2230.

22 Y. Xu, W. Chen, B. Yin and L. Huang: Agric. Mach. Technol. Ext. 9 (2004) 29.

23 GB/T 7975-2005: Paper and Board - Determination of Color (Diffuse Reflectance Method).

24 T. Luo: Study on the Characteristic Aroma and Quality Evaluation of Tea, Thesis of China Agric. Univ. (2009).

25 Z. Sun, J. Zhao, X. Zou and G. Li: J. Jiangsu Univ. 31 (2010) 139.

26 Q. Zhang, X. Wang and Y. Liu: Food Sci. 30 (2009) 284.

27 M. Kashaninejad, A. A. Dehghani and M. Kashiri: J. Food Eng. 91 (2009) 602.

28 A. M. Nederkassel, M. Daszykowski, D. L. Massart and Y. V. Heyden: J. Chromatogr. A 1096 (2005) 177. 